\title{
PVP2016-63848
}

\section{DRAFT: MICROFLOW LEAKAGE THROUGH THE CLEARANCE OF A METAL-METAL SEAL}

\author{
Ali A. Anwar* \\ WEIR Advanced Research Centre \\ Mechanical and Aerospace Engineering \\ University of Strathclyde \\ Technology and Innovation Centre \\ 99 George St, Glasgow, G1 1RD \\ ali.anwar@strath.ac.uk
}

\author{
Konstantinos Ritos \\ Yevgen Gorash \\ William Dempster \\ David Nash \\ Mechanical and Aerospace Engineering \\ University of Strathclyde, \\ James Weir Building \\ 75 Montrose St, Glasgow, G1 1XJ \\ konstantinos.ritos@strath.ac.uk, \\ yevgen.gorash@strath.ac.uk, \\ william.dempster@strath.ac.uk \\ d.nash@strath.ac.uk
}

\begin{abstract}
The motivation behind this study is to simulate high pressure gas flow through the clearance between a valve seat and disc when in a closed position using a representative model. This leakage phenomenon is common in metal-to-metal seal pressure relief valves. As a pressure relief valve reaches the set pressure, it is known for the leakage to increase. The representative model that we studied is of an ideal-gas flow through a 2D microchannel in the slip flow regime. We used a laminar continuum flow solver which solved the mass, momentum and energy equations. In addition, we applied low pressure slip boundary conditions at the wall boundaries which considered Maxwells model for slip. The channel height was varied from $1 \mu \mathrm{m}$ to $5 \mu \mathrm{m}$ while the length remained at $1.25 \mathrm{~mm}$, this means the length to height ratio varied from 1250 to 250 . Inlet pressure was varied from a low pressure $(0.05 \mathrm{MPa})$ to a high pressure (18.6 MPa), while the outlet remained constant at atmospheric. The calculated mass flow rate is compared to an analytical solution giving very good agreement for low pressure ratios and high length to height ratios.
\end{abstract}

${ }^{*}$ Address all correspondence to this author.

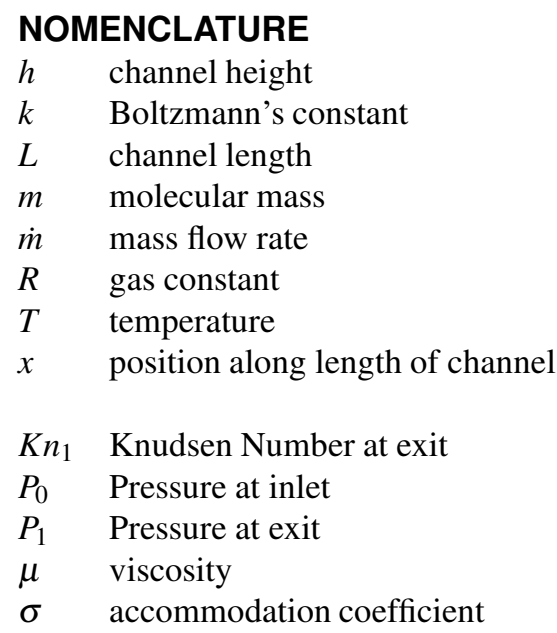

\section{INTRODUCTION}

Pressure Relief Valve (PRV) leak tightness guarantee provided by manufacturers is of great importance to both consumers and manufacturers. A consumer would want to ensure the leak tightness is guaranteed to safeguard working conditions. To gurantee the leak tightness, a PRV must comply with standards such as API 527: Seat Tightness of Pressure Relief Valves [1] and 
dependant on region the equivalent standard would be used i.e. British Standard-BS EN ISO 4126-1:2013 [2]; ASME StandardASME PTC 25-2014 [3], etc. As a PRV reaches its set pressure the leakage rate increases. Therefore having the ability to design according to standards and reduce the leakage of a PRV allows valve manufacturers to create market competitive products.

Primarily, this paper aims to allow designers of PRV's to consider fluid flow through a channel created between the valve seat and disc. The channel considered in this study is represented as a microchannel, while the fluid flow conditions are in the regime of slip flow. Due to the nature of the problem, the findings in this study and modelling approach can be extended for other geometric models to account for other devices or high pressure fields such as pipe sealing or gaskets.

The channel is modelled as 2D (as shown in Figure 1) being representative of a PRV seat and disc contact length $(L)$. The channel (or gap) can be attributed to an average spacing between the seat and disc contacting surfaces with respect to surface roughness, waviness and/or form i.e. the finish quality of the metal-to-metal contacting surfaces [4].

The first analysis in this study focuses on the effects of changing the inlet pressure, $P_{0}$, from $1 \mathrm{MPa}$ to $18.6 \mathrm{MPa}$ over a fixed channel height $(h)$ of $1 \mu \mathrm{m}$ and length of $1.25 \mathrm{~mm}$ $(L / h=1250)$ using CFD. For this study the internal pressure is higher than the external pressure, $P_{1}$ (remaining at atmospheric), consequently driving the gas to the outer extremity of the seat and disc length. This allows examination of the pressure profile across the 'Pressure Profile Line' (see Figure 1) which is the midline between seat/disc contact and how well it fairs with analytical equations. The channel height is then changed to $5 \mu \mathrm{m}$ $(L / h=250)$ and the mass flow rate is analysed.

The second analysis focuses on varying the channel ratio, $L / h$, from 1250 to 250 for the highest pressure of $18.6 \mathrm{MPa}$ and seeing how this effects the mass flow rate. This is to distinguish at which point the analytical equations are no longer appropriate.

The pressure distribution across the seat/disc length and the mass flow rate is calculated using the CFD solver, ANSYS Fluent v16.1 and compared against analytical models. The analytical models used were formulated by the well known equations/formula of Arkilic et al [5] that takes into account rarefraction in calculating the pressure distibution and mass flow rate at the exit across a parallel channel. A more recent analytical equation by Chong [6] for chocked flow is also compared and assessed.

\section{Background}

Arkilic et al's [5] analytical Eqn. (1) of the modified NavierStokes equation has shown good agreement with the classical microchannel pressure profile experiment conducted by Pong et al [7]. Arkilic et al's own experimental work showed good agreement with Eqn. (1).

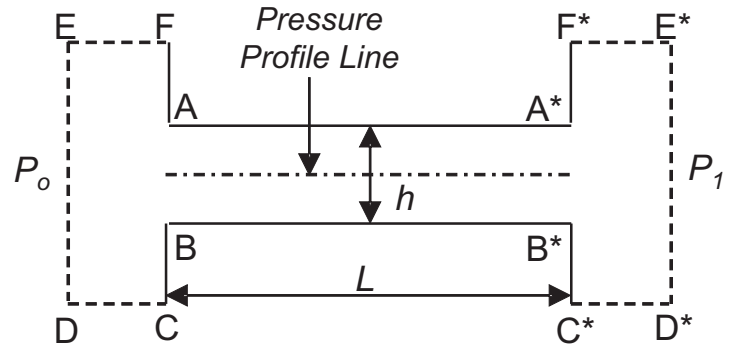

FIGURE 1. Sketch of computational regions

$$
\begin{aligned}
\frac{P(x)}{P_{1}} & =\left\{a^{2}-b\left(\frac{x}{L}\right)\right\}^{\frac{1}{2}}-6 K n_{2} \\
\text { where; } \quad a & =\left(6 \mathrm{Kn}_{2}+\frac{P_{0}}{P_{1}}\right) \\
b & =\left[\left(\frac{P_{0}^{2}}{P_{1}^{2}}-1\right)+12 \mathrm{Kn}_{2}\left(\frac{P_{0}}{P_{1}}-1\right)\right]
\end{aligned}
$$

Other authors have also shown good agreement with Eqn. (1) and Pong et al's experiment by conducting similar studies using up to pressure ratios $\left(P_{0} / P_{1}\right)$ of 3.95 (as shown in Table 1) for subsonic flows.

In past work, the channel length and height ratio (and Knudsen number) is such that the $L / h>1000$. This means that the poiseuille flow equation is satisfied since it is assumed that the $L / h \gg 1$ and, low Mach numbers, isothermal flow is considered between two parallel plates [8]. Using Arkilic et al's [5] equation the mass flow rate can be calculated (which accounts for rarefaction) using:

$$
\dot{m}=\frac{h^{3} P_{0}^{2}}{24 \mu R T}\left[P_{0}^{2}-P_{1}^{2}+12 \frac{2-\sigma}{\sigma} K n_{1} P_{1}\left(P_{0}-P_{1}\right)\right]
$$

Equation 2 has however been used for $L / h<1000$ by Chong, who considers chocked conditions for subsonic flow. Chong accounts for subsonic flow conditions using the equation below:

$$
\dot{m}=\frac{h^{3} P_{0}^{2}}{24 \mu R T}\left(1+12 \frac{(2-\mu)}{\mu} \frac{\sigma}{P_{1} h} \frac{16}{5} \sqrt{\frac{k T}{2 \pi m}}\right) .
$$

Chong creates this equation by assuming the pressure ratio $\left(P_{0} / P_{1}\right)$ tends to infinity for subsonic chocked flow conditions in the microchannel. This analytical equation is also compared in this study, however what stands out is that there are no terms to consider the effects of compressibility i.e. the change in density/temperature as the transition is made from subsonic to chocked conditions. This is discussed latter. 
TABLE 1. Selected authors who have conducted experiments and compared Eqn. (1) and Eqn. (2) for various pressure ratios $\left(P_{0} / P_{1}\right)$ and $L / h$ conditions.

\begin{tabular}{clll}
\hline Research Papers & $L / h$ & $P_{0} / P_{1}$ & $K n$ \\
\hline Pong et Al (1994) [7] & 2500 & $1.35-2.75$ & Slip Flow \\
Arkilic et Al (1997) [5] & $>5600$ & $1.2-2.5$ & Slip Flow \\
Zohar (2002) [9] & $\geq 4000$ & $2.5-3.95$ & Slip Flow \\
Chong (2006) [6] & $5-100$ & $1-32$ & Slip Flow- \\
\hline
\end{tabular}

\section{SIMULATION CONDITIONS AND METHOD}

Figure 1 shows the 2D sketch of the simulated microchannel with channel size, $h$, spanning from $\mathrm{AB}$ to $\mathrm{A} * \mathrm{~B} *$ with a fixed length, $L=1.25 \mathrm{~mm}$. AB is the inlet and $\mathrm{A}^{*} \mathrm{~B} *$ is the outlet of the microchannel. A buffer region is added either side of the inlet and outlet of the microchannel (zone CDEF and $\mathrm{C}^{*} \mathrm{D}^{*} \mathrm{E}^{*} \mathrm{~F}^{*}$ respectively). If the buffer regions were not present then it would mean analysing results at the boundary condition of the inlet and the outlet, with no guarantee of the model flow being fully developed at those points.

For the CFD simulations, it is assumed that the temperature at the walls and of the laminar flow of gas is $295 \mathrm{~K}$ and the fluid is an ideal gas being air. The walls $\mathrm{AA}^{*}$ and $\mathrm{BB} *$ have also a low pressure boundary slip condition applied which allows the Maxwells model for velocity slip and temperature change to be considered $(0.01<K n<0.1)$ [10].

The CFD simulations have been conducted in ANSYS Fluent v16.1 for these studies. A meshing example of the inner buffer region for channel height of $1 \mu \mathrm{m}$ is shown in Figure 2. As shown, the meshing becomes more concentrated as we travel towards the inlet of the microchannel. Similarly the walls $A B$ and $\mathrm{A} * \mathrm{~B} *$ have many layers/divisions with a bias towards the wall. This allows the velocity and temperature change across the wall to be captured with better precision.

The first results focus on a channel height of $1 \mu \mathrm{m}$ $(L / h=1250)$ with the pressure inlet varied from $1-18.6 \mathrm{MPa}$. Specifically, pressure inlet $\left(P_{0}\right)$ values of $1,5,10,15$ and $18.6 \mathrm{MPa}$ are analysed. After which the mass flow rate is found at the outlet, $\mathrm{A}^{*} \mathrm{~B}^{*}$, for a channel height of $1 \mu \mathrm{m}$ and $5 \mu \mathrm{m}$ $(L / h=250)$. The CFD simulation is compared against Eqn. 1 to see how well the analytical model fairs against the CFD. Similarly the mass flow rate calculated via the CFD is compared to Eqn. 2 and Eqn. 3.

The second set of results focus on changing the $L / h$ ratio from $1250-250$, specifically $L / h$ values of $1250,1136,1042$, $961.5,892.9,833.3,625,416.7,312.5$ and 250 and measuring the mass flow rate. This time the focus is primarily on seeing the difference between the analytical Eqn. 2 and the CFD calculations.

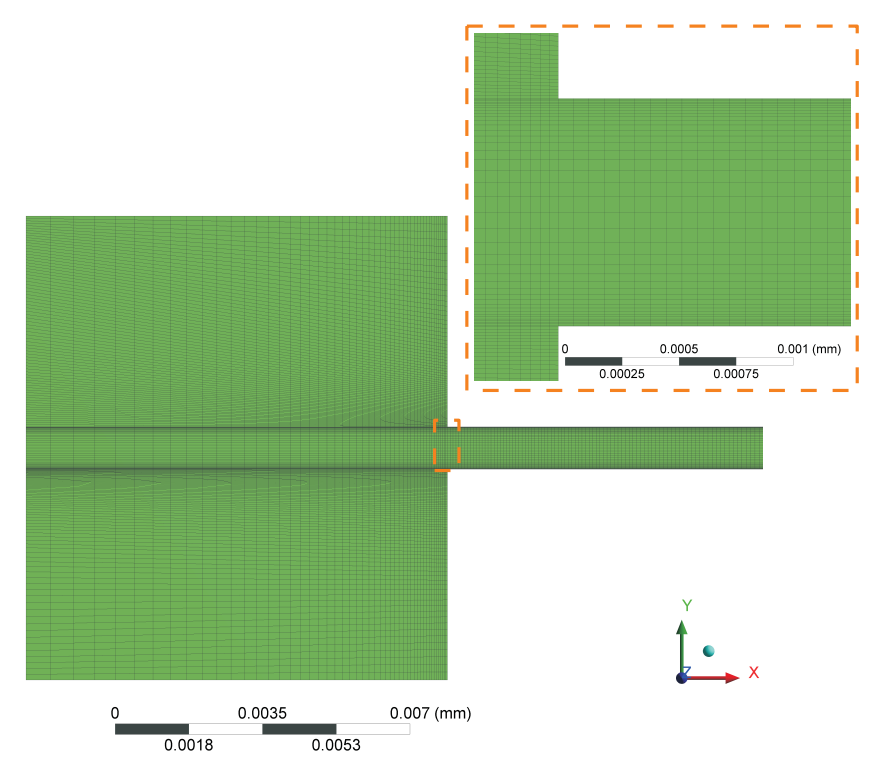

FIGURE 2. Mesh of inner buffer region where $\mathrm{h}=1 \mu \mathrm{m}$

\section{RESULTS AND ANALYSIS}

The first set of results shown in Figure 3 are of the pressure profile across the seat length, $L$.

As it can be be seen there is good agreement when $P_{0}$ up to 5 $\operatorname{MPa}\left(P_{0} / P_{1}=49.3\right)$ between the CFD simulation and the analytical model. There is a very slight deviation between the $5 \mathrm{MPa}$ which begins at about $0.88 \mathrm{~mm}$ along the channel length. Referring to Figure 4, this is most likely because the Mach number at the exit reaches 1 for this condition meaning that compressibility effects on the gas need to be considered. This measurement is however taken at the centreline of the channel (Pressure Profile Line). As we move toward either wall of the channel the Mach number decreases.

As the inlet pressure is ramped up to $18.6 \mathrm{MPa}$ the deviation between the analytical Eqn. (1) and simulated results becomes more prevalent for each pressure increment. Only the final $2 \mu \mathrm{m}$ of the channel length show chocking conditions. This is due to the compressibility of the gas becoming more apparent and not being considered in the analytical model. Equation 1 was originally formulated for Micro-Electro-Mechanical Systems (MEMS) equipment [8] which require low pressure ratios, sub-sonic flow conditions, therefore this deviation between the CFD and analytical results for $P_{0} / P_{1} \geq 49.3$ is not surprising.

The next set of results of interest are of the mass flow rate at the exit of the microchannel. It is with the mass flow rate we can attribute leakage. So, for $h=1 \mu \mathrm{m}(L / h=1250)$ and $h=5 \mu \mathrm{m}$ $(L / h=250)$ the mass flow rate is calculated using Eqn. (2) and Eqn. (3) and comparing it against the CFD simulation for the same pressure range as before. The results are shown in Figure 5 and Figure 6.

It can be seen for the $\mathrm{L} / \mathrm{h}=1250$, the mass flow rate for the CFD simulation and the analytical equations compare well. While for the $\mathrm{L} / \mathrm{h}=250$ it is clear that after $5 \mathrm{MPa}$ the analytical equations tend toward an exponential direction. While the CFD 


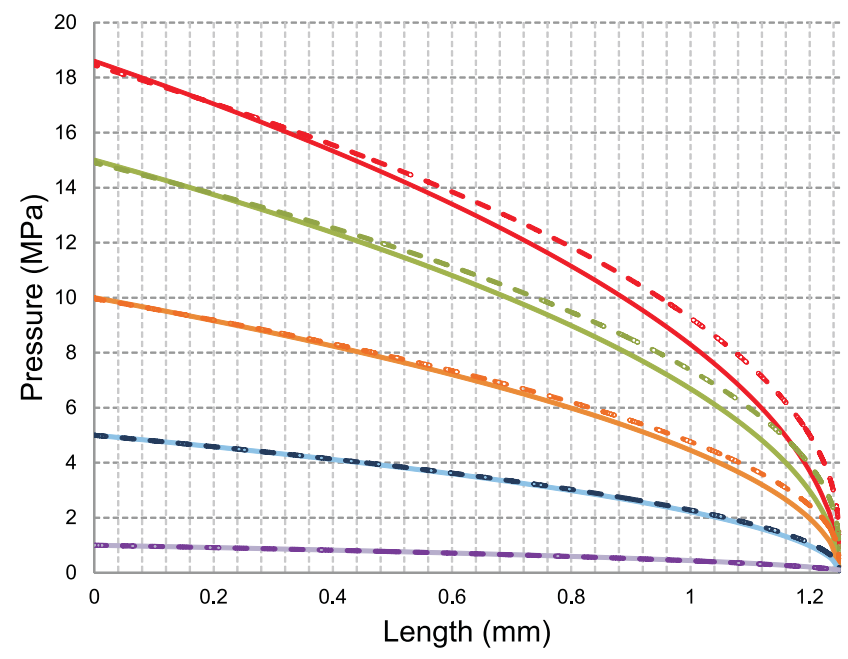

Arkilic et al Eqn. (1)-18.6MPa - - ANSYS Fluent
Arkilic et al Eqn. (1)-15MPa - - ANSYS Fluent
Arkilic et al Eqn. (1)-10MPa - - ANSYS Fluent
Arkilic et al Eqn. (1)-5MPa - - ANSYS Fluent
Arkilic et al Eqn. (1)-1MPa - - ANSYS Fluent

FIGURE 3. Graph of Pressure profile versus length for $\mathrm{h}=1 \mu \mathrm{m}$

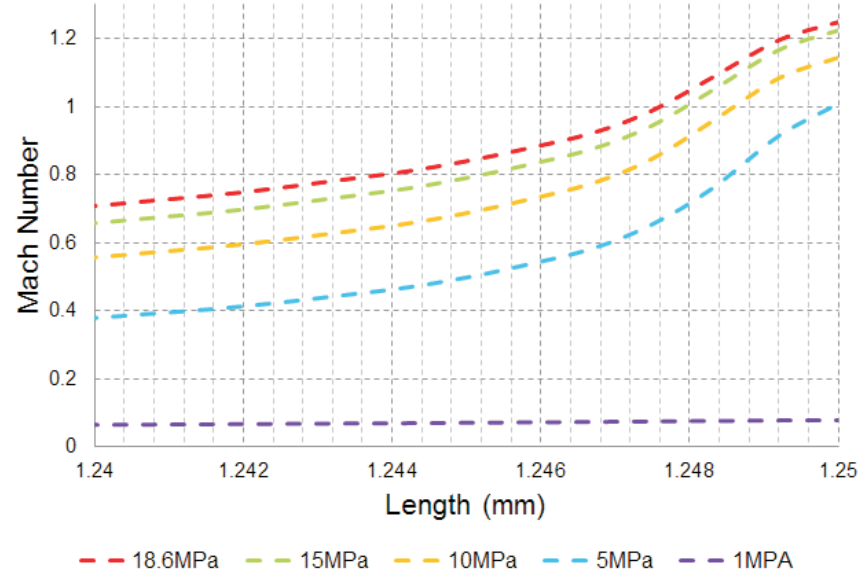

FIGURE 4. Mach Number versus channel length (last $0.1 \mathrm{~mm}$ ) for $\mathrm{h}=1 \mu \mathrm{m}$

solver follows a straight line trend.

Equation 3 shows negligible difference in comparison with Arkilic et al's Eqn. (2). As discussed earlier, it is known that at $5 \mathrm{MPa}$ the Mach number was found to be 1 at the exit, therefore the percentage difference found at $5 \mathrm{MPa}$ is $51 \%$ between Eqn. 3 and the CFD simulation. Chong [6] has stated to find a difference of $45 \%$ between the DMCS and Eqn.(3) for a Mach number of 0.9 at the exit due to a pressure ratio of 32 . Therefore, there is good correlation here between Chong's study and this CFD study. It should be noted that Chong used a much lower $L / h$ ratio of 5. It would be worthwhile to simulate the exact same experiment conducted by Chong and compare it using CFD to see how well CFD fairs with the Direct Simulation Monte Carlo

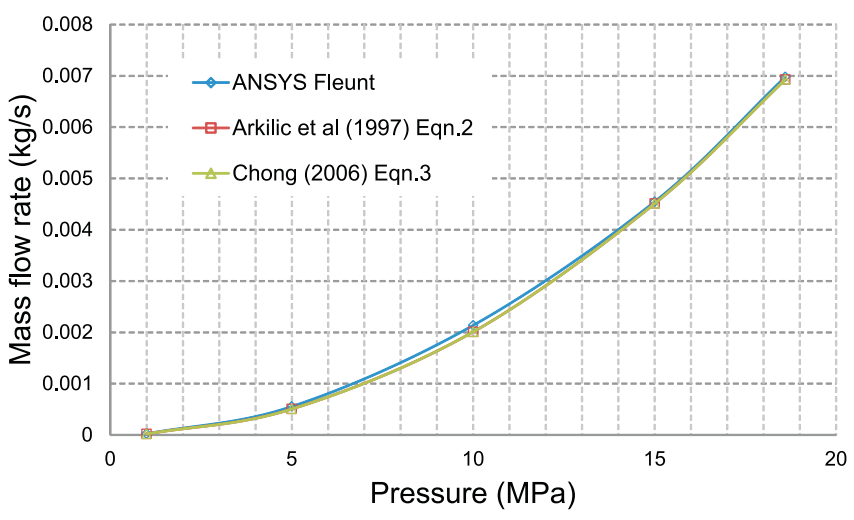

FIGURE 5. Graph showing the effect of $P_{0}$ change from $1 \mathrm{MPa}-$ $18.6 \mathrm{MPa}$ versus the mass flow rate for a channel of $\mathrm{h}=1 \mu \mathrm{m}$

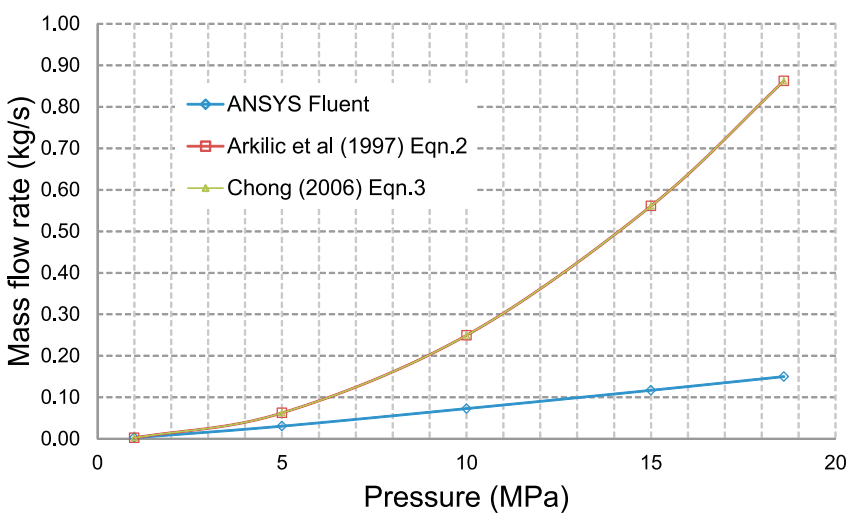

FIGURE 6. Graph showing the effect of $P_{0}$ change from 1 MPa-18.6 MPa versus the mass flow rate for a channel of $\mathrm{h}=5 \mu \mathrm{m}$

(DSMC) method for Chong's scenario.

However, this good correlation does not detract from the exponential difference between the CFD simulation and the analytical equations as seen in Figure 6. As stated earlier Eqn.(2) created by Arkilic et al is only applicable for subsonic flows and therefore does not consider the effects of compressibility. Also it is based on Poisellue flow which assumes that $L / h>1000$. To analyse the effects of $L / h$ the previous analysis is extended to find the mass flow rate for a inlet pressure of $18.6 \mathrm{MPa}$ for $L / h$ ratios of 1250, 1136, 1042, 961.5, 892.9, 833.3, 625, 416.7, 312.5 and 250. The results are plotted in Figure 7.

Up to a $L / h$ ratio of 1000 there is a maximum difference of $16 \%$ between the CFD and analytical model. When $L / h>1000$ there is another exponential increase in the difference between the CFD and analytical calculations increasing all the way up to $475 \%$. This shows that the compressibility effects of the gas are likely to be a much more dominating effect. To conclusively know if the CFD solver is correctly calculating the pressure profile and mass flow rate, experiments would be required, replicating the a $L / h<1000$ and high pressure ratios which are capable of chocked flow conditions. Also the DSMC method should 


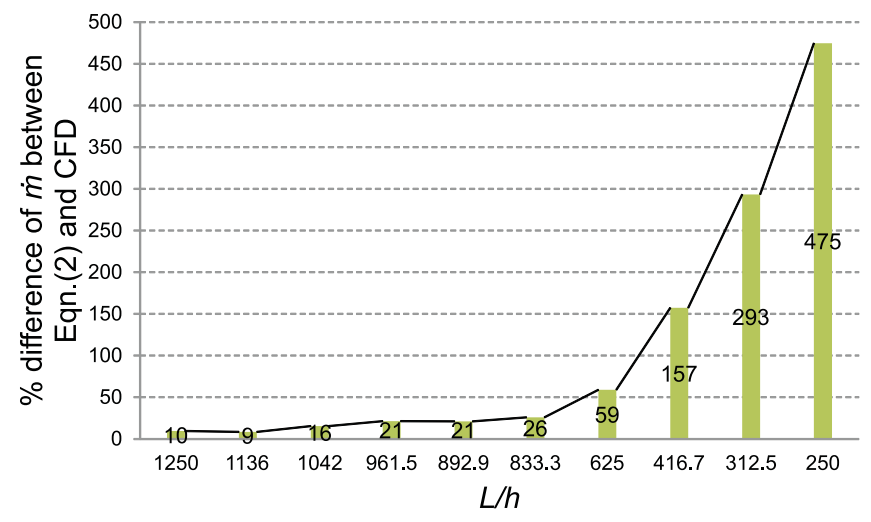

FIGURE 7. Graph showing the effect of changing L/h from 1250250 versus the percentage difference of the mass flow rate between the Arkilic et al analytical Eqn. (2) and the CFD solver (ANSYS)

be ran in parallel similar to Chong's analysis method (excluding Eqn. 3).

\section{CONCLUSIONS}

This study has shown that for low pressure ratios of $P_{0} / P_{1}<49.3$ where the flow conditions are not able/capable to create chocked flow conditions in a microchannel and with a $L / h>1000$, the analytical equations created by Arkillic are appropriate since they disregard the effect of compressibility.

However, for high pressure ratios of $P_{0} / P_{1} \geq 9.87$ where the flow conditions create a chocked flow in a microchannel and with a $L / h<1000$, the analytical equations created by Arkillic and Chong are not appropriate since they disregard the effect of gas compressibility. Instead CFD and DSMC simulations for this condition are likely to be of greater reliance since they do take into consideration the effects of compressibility of the gas as it reaches the outlet of the microchannel. Experimental studies would be required to validate the CFD and DSMC simulations.

\section{ACKNOWLEDGMENT}

Thanks go to the authors who have dedicated there time and great scientific contributions to allow this study to be conducted in the first place.

Thanks also goes to Craig White from the University of Glasgow for his input and advice on this topic.

Lastly, much thanks to the WEIR organisation in particular: Alan Bickley, Stephane Charrier, Fabrice Courdavault, Alan Stewart and Ian MacQueen for their guidance and advice.

\section{REFERENCES}

[1] API, 2014. Seat Tightness of Pressure Relief Valves. No. 527 in API Standard. American Petroleum Institute, Washington, USA.

[2] BSI, 2013. Safety devices for protection against excessive pressure. Safety valves. No. BS EN ISO 4126-1:2013 in
British Standard. The British Standards Institution, London, UK.

[3] ASME, 2014. Pressure Relief Devices - Performance Test Codes. No. ASME PTC 25-2014 in An American National Standard. The American Society of Mechanical Engineers, New York, USA.

[4] Anwar, A., Gorash, Y., Dempster, W., and Hamilton, R., 2015. "Literature research in relevant fields to understand pressure relief valve leak tightness in a static closed state". Procedia Engineering, 130, pp. 95 - 103.

[5] Arkilic, E. B., Schmidt, M., Breuer, K. S., et al., 1997. "Gaseous slip flow in long microchannels". J. of Microelectromechanical Systems, 6(2), pp. 167-178.

[6] Chong, X., 2006. "Subsonic choked flow in the microchannel”. Physics of Fluids, 18(12), pp. 127104-1-127104-5.

[7] Pong, K.-C., Ho, C.-M., Liu, J., and Tai, Y.-C., 1994. "Non-linear pressure distribution in uniform microchannels". ASME-PUBLICATIONS-FED, 197, pp. 51-51.

[8] Karniadakis, G. E., Beskok, A., and Aluru, N., 2006. Microflows and nanoflows: fundamentals and simulation, Vol. 29. Springer Science \& Business Media.

[9] Zohar, Y., Lee, S. Y. K., Lee, W. Y., Jiang, L., and Tong, P., 2002. "Subsonic gas flow in a straight and uniform microchannel". Journal of fluid mechanics, 472, pp. 125-151.

[10] ANSYS $^{\circledR}$ Help, 2015. Fluent // Theory Guide // 7. Species Transport and Finite-Rate Chemistry // 7.2.3. Slip Boundary Formulation for Low-Pressure Gas Systems, Academic Research 16.1.0 ed. ANSYS, Inc., Canonsburg (PA), USA. 